\title{
Summarizing scrambled stories
}

\author{
WALTER KINTSCH, THEODORE S. MANDEL, and ELY KOZMINSKY \\ University of Colorado, Boulder, Colorado 80309
}

\begin{abstract}
Subjects read stories 1,400 words long and wrote 60- to 80-word summaries of these stores. Reading time was either unrestricted or limited, and the stories were either presented in natural order or with their paragraphs scrambled. Reading times were longer for the scrambled stories, but the same kind of summaries were produced whether stories were presented in natural or scrambled order. When a well-structured story was used, judges were unable to distinguish the two types of summaries and rated the adequacy of summaries based upon the scrambled version about as high as that of summaries based upon normal stories. For a less well-structured story, judges could differentiate between the summaries from natural and scrambled stories. These results demonstrate the effectiveness of the story schema which permits subjects to comprehend scrambled stories by reorganizing them.
\end{abstract}

Text comprehension involves organizing the text in some way and relating the new information provided by the text to knowledge already available. This process of organization and apperception is facilitated when it is controlled and guided by a schema. Text schemata are general knowledge structures that abstract the conventions and principles observed by any given culture in constructing particular types of text. The best understood text schema is the one for stories. Bartlett (1932) was the first psychologist to explore the involvement of schemata in story comprehension. Recently, the study of story comprehension has again become an active research topic among psychologists (Rumelhart, 1975; Schank, 1975; Thorndyke, 1977), and in some of this research the concept of a story schema plays a crucial explanatory role (Kintsch \& van Dijk, 1975; Mandler \& Johnson, 1977; van Dijk \& Kintsch, in press). The present paper provides an experimental demonstration of how the schema guides comprehension under unfavorable experimental conditions, that is, when the stories presented to subjects are scrambled.

In order to understand the theoretical implications of the experiment reported below, at least an outline of a theory of story comprehension is required. We assume that comprehension involves, inter alia, the construction of a propositional text base representing the meaning of a text. When the text is sufficiently long, however, this propositional base must be organized into a sequence (or possibly a hierarchy) of subunits. This organization has been called the macrostructure of a text. Each subunit consists of a partially ordered set of propositions, subordinated to a label for that unit, called a macrostructure proposition. A theory of comprehension must specify: (1) how the division into subunits is achieved, and (2) how the macro-

This research was supported by Grant 15872 from the National Institute of Mental Health. proposition for each subunit is derived from the text. It is the first of these processes, the organization into subunits, that can be guided by a schema. Essentially, what we are saying is that the organization of a story is facilitated when the comprehender knows how such a text is usually organized. Thus, the story schema consists of certain kinds of knowledge that readers have about the nature of stories. Specifically, the following points are important in our culture.

(1) The story must have one (or a few) principal character (the hero) who appears in most of it. The roles of the other actors are defined with respect to the hero.

(2) The events in the story must be causally and temporally related.

(3) The story consists of a sequence of episodes, each episode having a tripartite internal structure, usually denoted by the terms exposition, complication, and resolution. Each of these story categories may in turn consist of one or more episodes. This recursiveness permits a variety of structures, while still retaining the basic simple form.

(4) It is the function of an exposition to permit the reader to set up a stable frame to interrelate the propositions of that episode and to infer missing ones. The complication introduces a new frame. The relationship between the frame of the exposition and complication is a subtle one. On the one hand they cannot be unrelated, but neither can they be too closely related. The complication must provide something interesting or remarkable, which means that in the frame change from exposition to complication there always must be an element of surprise. The resolution, on the other hand, either retains the frame of the complication or returns to the exposition frame. The events of the resolution are a direct consequence of the complication.

(5) The terms exposition, complication, and resolution should not be taken too literally. Frequently, 
for instance, the "exposition" of an episode describes a state of affairs where the hero desires a certain goal, but the achievement of that goal is blocked ("complication"); this is followed by an unexpected restructuring ("resolution") of the situation which removes the block from the goal. Terms with less surplus meaning might be preferable [e.g., exposition, happening, and consequence, or state, change of state (action), and new state].

Not any text describing any event is, therefore, a story. For instance, describing how Mary waters the tomatoes in her garden is not a story in the sense used here. On the other hand, there are many stories that are much more complicated structurally. Indeed, a good short story derives much of its literary merit from the way the author plays with the simple story schema of his readers (with flashbacks, changes in viewpoint from one character to another, and the like).

According to the theory outlined above, the use of the reader's knowledge is crucial in comprehending a story. As in all text comprehension, the reader must use his general knowledge about the world in order to understand a story. This general knowledge is organized in terms of units called frames, and in story comprehending the reader calls up from his memory a network of instantiated frames that allows him to infer material needed for the comprehension of the story but not directly expressed in the text.

We are concerned here specifically with one kind of general knowledge, namely, knowledge about the structure of stories, that is, the story schema. If a subject reads a story that is structured in accordance with his schema, it should not matter greatly if the paragraphs of the story are presented out of order. The story schema assigns a place and a function to each, and the reader should be able to reorganize the scrambled story in his mind. Both the story schema and the subject's knowledge about what is likely to follow what in the story (or in the world) are involved in this reconstruction. The prediction of the theory that will be tested here is, therefore, that summaries written after reading a story in scrambled order are as good as summaries written after reading the story in its natural order.

Note that we are scrambling whole paragraphs, not sentences. Scrambling the order of sentences in a paragraph destroys intraparagraph cohesiveness which integrates the sentences into a text. Pronoun referents for definite articles cannot be determined (see Rosenberg \& Lambert, 1974). Other cohesion elements, such as lexical cohesion and conjunctive relations (Halliday \& Hasan, 1976), that contribute to a paragraph's cohesion may also be disrupted. However, the same difficulties are greatly reduced when paragraphs rather than sentences are scrambled. Usually, few cohesive elements cross paragraph boundaries and their recovery can be facilitated by applying the story schema (if the story conforms to this schema).

If given enough time, subjects' comprehension of stories in which the paragraphs are scrambled should be as good as that for stories presented in normal order. This prediction was confirmed in an experiment reported by Kintsch and van Dijk (1975; see also van Dijk \& Kintsch, in press). They had subjects read two stories (in fact, the same stories as were used in the experiment to be reported below), either in normal order or with their paragraphs scrambled. Subjects then summarized these stories in 60 to 80 words. An analysis of the propositions used in these summaries revealed that subjects agreed among themselves equally well whether they had read the scrambled or the normal stories.

The present experiment replicates and extends these results. It differs from the previous study in two ways. First, more direct methods of data analysis were used. In Experiment 1, the quality of the summaries produced was evaluated by judges who tried to distinguish between normal and scrambled stories on the basis of the way subjects had summarized them. In Experiments 2 and 3, the summaries from natural and scrambled stories were rated for their acceptability as "good" summaries of these stories. Second, the scrambling procedure was used to find out whether macrostructures are formed as an integral part of the comprehension process, or only when the task demands it (e.g., when the subject is asked to summarize a story). If macrostructures are formed as part of the initial comprehension process, this process should be more difficult (and take longer) for scrambled stories than for natural stories. Once the subject has read a scrambled story, however, it should take him no longer to summarize it than a normal story, and the summary should be as good as that of a normal story.

These predictions hold for the case when subjects are given unlimited time to read the story. But if reading time is sufficiently restricted, subjects who are trying to comprehend the scrambled stories might not be able to finish the process of reorganizing them, and hence should be able to form only a partial macrostructure. This should be reflected in a deterioration in the quality of their summaries. If judges who have never read the stories can discriminate between summaries from normal and scrambled stories, we can conclude that the scrambled stories were not successfully restructured; if the judges fail to make this discrimination, we can conclude that the scrambled stories were comprehended as well as normal stories. This is the result predicted for the free reading time condition. When subjects are not allowed much time to read the scrambled stories, they should be less successful in reconstructing the macrostructure of the story, and hence the judges should be able to discriminate between summaries from scrambled stories and summaries from normal stories. 
Table 1

The Macrostructure of "Simona"

Episode 1

Exposition:

Simona and Pasquino are lovers.

Complication:

Episode 2

Exposition:

They meet in a garden.

Complication:

Pasquino is poisoned by a sage leaf and dies. Resolution:

Simona is accused of poisoning Pasquino.

Episode 3

Exposition:

The judge makes Simona reenact the poisoning. Complication:

Simona is poisoned by the sage and dies. Resolution:

A toad is discovered under the sage bush.

Resolution:

The lovers are united in death.

Moral

Happy souls!

\section{EXPERIMENT 1}

\section{Method}

Subjects. All subjects were students from the University of Colorado who were able to type and who participated in the experiment as partial fulfillment of a course requirement. The experimental sessions lasted up to $2 \mathrm{~h}$.

Twenty-four subjects participated in the free reading condition, and 28 in the restricted reading condition. A further group of 21 subjects served as raters.

Stories. Two stories were selected from a modern (1972) translation of Boccaccio's Decameron, "Simona" (Story 7, fourth day), and "Rinaldo" (Story 2 , second day). The stories were both about 1,400 words long ("Rinaldo" had to be cut somewhat to reduce it to that length). "Simona" was divided into 13 paragraphs and "Rinaldo" into 15 , which were numbered successively for identification.

Tables 1 and 2 show the macrostructure of the two experimental stories in outline form. Both stories are simple in structure and conform closely to the story schema. However, there are important differences between the two stories. For "Simona," all three episodes are tightly interrelated in terms of place, time, and most importantly, causality. Episode 2 is a direct consequence of the exposition of the story: The lovers arrange a clandestine meeting in a garden. There the boy, Pasquino, suddenly dies, and again as a direct consequence, Simona is accused of poisoning him. The trial follows, which in turn causes Simona's death, and all this finally leads up to reunion in heaven. Thus, parts of the story that are presented out of their natural order are easily rearranged. This is not the case, at least not to that extent, for the other story, "Rinaldo." The seduction episode follows the robbery episode in quite an arbitrary way (though it is not entirely unmotivated in the story). Rearranging the two episodes would do violence to the original intent of the story, but it would not completely destroy it, so that if the story is presented in scrambled order, a reader might easily fail to rearrange it correctly. Thus, "Simona" conforms more closely to the story schema than "Rinaldo," which violates the requirement for strict causal connections between episodes.
We are, therefore, predicting different results for the two stories. The predictions made above were made for a tightly organized, well-structured story like "Simona." We would expect that they would not hold, or only partially hold, for "Rinaldo."

Procedure. In the free reading condition, subjects were given a typewritten copy of each story to read at their own pace. "Simona" was always presented first and "Rinaldo" second. A third story of comparable length and style was used for practice and warm-up. Thus, the experimental stories were always the second and third stories summarized in a session. Summaries were written immediately after reading each story. Subjects were told to write a summary of 60 to 80 words that "reproduces the most important events in the story." Subjects typed on a computer-controlled typewriter, with the text appearing on a screen before them. The computer also displayed the number of words written. Subjects were instructed in how to edit and revise their summaries. The reason for restricting the summaries in length was that preexperiments had shown that without such a restriction summaries differed in length so widely as to make meaningful comparisons between them problematic.

Half of the subjects read each story in normal order and the other half read each story with the paragraphs scrambled randomly. The same randomization was used for all subjects. Subjects were handed a mimeographed story and their reading time was recorded. They were not permitted to refer to the story while writing their summaries. Summary writing times did not include the time required to instruct subjects in the use of the computer-controlled typewriter. The experiment was conducted at the Computer Laboratory for Instruction in Psychological Research (CLIPR) of the University of Colorado.

In the restricted reading condition, subjects were allowed to read each story for only a fixed length of time, which was $58 \%$ of the average time taken for each story when reading times were unrestricted. This permitted subjects to read through each story without giving them much extra time.

In the rating phase of the experiment, subjects were given a set of 10 summaries in the form of computer printouts with the following instructions.

"This is a follow-up session for an experiment that we have done on reading, comprehending, and recalling stories. The stories we have used are from an old Italian story book. One is called 'Simona' and the other is 'Rinaldo.'

"In our experiment, the stories were read in two versions. Half of the students read the stories in the original form, called natural order, while the rest read the stories with all the paragraphs mixed up. This we called scrambled order stories.

"Everyone then wrote a short summary of the stories they

Table 2

The Macrostructure of "Rinaldo"

Episode 1

Exposition:

The merchant Rinaldo meets three men on his travels. Complication:

They discuss the efficacy of prayer with him, rob him, and leave him destitute. Resolution:

Episode 2 Exposition:

A lady shelters Rinaldo. Complication:

The lady seduces Rinaldo. Resolution:

Rinaldo leaves in the morning.

Rinaldo returns home and the robbers are hanged. 
read, and now we wish to see if there are any differences between the summaries for the natural order stories and the scrambled order stories.

"You will get four sets of 10 summaries each, two sets from each story. Half of the 10 summaries in each set are from natural stories, and the other 5 are from scrambled stories. When you get each set, read through all 10 summaries to get an idea of the story, then sort the summaries into two groups: ones that you think are from the natural stories, and the ones that you think come from the scrambled stories. You must finish with five summaries in each pile. Please ignore the ungrammaticalities in the summaries."

Of the four sets of summaries sorted by each subject, one contained summaries from Simona/free reading, one from Rinaldo/free reading, one from Simona/restricted reading, and one from Rinaldo/restricted reading. Each set contained five summaries from natural and five from scrambled stories. The 10 summaries in each set were selected randomly from the summaries stored in the computer files. They were printed out on separate sheets of paper, so that the subject could physically sort them out into two piles. The summaries which were sorted were edited for spelling and punctuation, and in a few cases such comments as "This story was out of order," or "This story did not make sense," were removed.

\section{Results}

Free reading. The reading times for "Simona" and "Rinaldo" were $8.30 \mathrm{~min}(.41 \mathrm{~min})$ and $8.10 \mathrm{~min}$ (.46 min), respectively (with the standard errors given in parentheses). This difference was not significant statistically $[\mathrm{t}(23)<.1]$.

The mean reading time for the stories in natural order was $7.34 \mathrm{~min}$ ( $\mathrm{SE}=.33 \mathrm{~min}$ ) and for scrambled stories, $9.05 \mathrm{~min}(\mathrm{SE}=.46 \mathrm{~min})$. This difference was highly reliable statistically $[\mathrm{t}(23)=6.14, \mathrm{p}<.001]$.

Average writing times were $11.04 \mathrm{~min}$ for stories that had been read in their natural order and $9.43 \mathrm{~min}$ for stories that had been read in the scrambled order. Thus, there is no evidence at all that summarizing scrambled stories takes longer than summarizing normal stories, though it does take longer to read such stories.

Raters could not tell which summaries were written from the scrambled version and which were written from the normal version of "Simona." On the average they sorted $49 \%$ of the summaries correctly, which is very close to chance $\left[\chi^{2}(20)=11.2\right]$. Sorting performance improved somewhat for "Rinaldo," with $64 \%$ of the summaries correctly classified. This is significantly different from chance $\left[\chi^{2}(20)=48.0, p=.001\right]$.

Restricted reading. The mean writing times for summarizing normal and scrambled stories were $9.81 \mathrm{~min}(\mathrm{SE}=.76 \mathrm{~min})$ and $10.06 \mathrm{~min}(\mathrm{SE}=.80 \mathrm{~min})$, respectively. Thus, subjects took about equally long to summarize normal and scrambled stories.

Raters managed to sort $52 \%$ of the summaries of "Simona" correctly, which is not different from chance $\left[\chi^{2}(20)=11.2\right]$. For "Rinaldo," on the other hand, the summaries written after reading the scrambled stories could be discriminated from the normal ones with an accuracy of $73 \%\left[\chi^{2}(20)=53.2, p<.001\right]$.

The predicted deterioration in the quality of summaries for scrambled stories when reading time was restricted occurred only for "Rinaldo," while "Simona" was correctly comprehended even when not much time was allowed for it. Overall, the number of summaries correctly classified as normal or scrambled were higher when reading time was restricted $(62 \%)$ than when it was free $(56 \%)$, but this difference did not reach statistical significance $[\mathrm{t}(42)=1.46, \mathrm{p}=.072]$.

\section{EXPERIMENT 2}

The sorting analysis has, in general, supported our predictions. The well-structured story summaries written from normal and scrambled texts could not be discriminated at all, while judges were somewhat more successful with the loosely structured story. On the other hand, the predicted deterioration of the summaries with reading time could not be demonstrated with the sorting method, though a statistically nonsignificant trend in the expected direction was obtained. This raises the problem that the sorting task may not be sensitive enough to detect the qualitative differences among the summaries of the two stories. Therefore, we decided to replicate the experiment with a different judgmental task: Subjects were asked to rate the acceptability of the summaries on a scale ranging from "good" to "poor." This task is not quite as constrained as the sorting task, and we hoped it would be somewhat more sensitive in discriminating between good and bad summaries.

\section{Method}

Subjects. Forty undergraduate students participated in partial fulfillment of a course requirement.

Materials. The summaries of "Simona" and "Rinaldo" obtained in the first experiment served as the rating material.

Procedure. Twenty subjects were randomly assigned to each of two groups. One group rated summaries from the free reading condition, and the other group worked with summaries from the restricted reading condition.

The subjects received the same background information about the source of the summaries as the subjects in the sorting experiment, except that they were not told anything about the scrambled and normal presentation orders.

Raters first read one of the stories; when they had done so, they put it away and rated a random sample of 10 summaries of that story on a 7-point scale. The endpoints of the scale were labeled: 1, "very poor summary" and 7, "very good summary." The same procedure was then repeated with the second story. The order in which subjects worked with the two stories was counterbalanced. Each set of 10 summaries contained 5 summaries based upon the natural version of the story and 5 from the scrambled version. The same sample sets were used as in Experiment 1.

\section{Results}

Table 3 summarizes the rating data for each story under the four experimental conditions. An analysis of variance with the factors reading time (free vs. restricted), story (Simona vs. Rinaldo), and condition (normal vs. scrambled) was performed with these data. 
Table 3

Mean Ratings of Summaries

\begin{tabular}{|c|c|c|c|c|c|c|c|c|}
\hline & \multicolumn{4}{|c|}{ "Simona" } & \multicolumn{4}{|c|}{ "Rinaldo" } \\
\hline & \multicolumn{2}{|c|}{ Free } & \multicolumn{2}{|c|}{ Restricted } & \multicolumn{2}{|c|}{ Free } & \multicolumn{2}{|c|}{ Restricted } \\
\hline & Natural & Scrambled & Natural & Scrambled & Natural & Scrambled & Natural & Scrambled \\
\hline $\begin{array}{l}\text { Experiment } 2 \\
\text { Experiment } 3\end{array}$ & $\begin{array}{l}3.86 \\
3.92\end{array}$ & $\begin{array}{l}3.60 \\
3.67\end{array}$ & $\begin{array}{l}3.66 \\
3.05\end{array}$ & $\begin{array}{l}2.70 \\
2.45\end{array}$ & $\begin{array}{l}4.11 \\
4.70\end{array}$ & $\begin{array}{l}3.00 \\
3.43\end{array}$ & $\begin{array}{l}3.98 \\
3.78\end{array}$ & $\begin{array}{l}2.39 \\
2.27\end{array}$ \\
\hline "Ideal" & 4.95 & & & & 4.78 & & & \\
\hline
\end{tabular}

The main effect of stories was not significant $(F<1)$, but normal summaries received higher ratings than scrambled summaries $[F(1,38)=75.00, p<.001]$ and free reading time summaries were rated higher than restricted reading time summaries $[F(1,38)=4.84$, $p=.034]$. These main effects are, however, qualified by two significant interactions. Restricting reading times made a difference only with scrambled stories, and not when the stories were in their natural order $[F(1,38)=6.80, \quad p=.013]$. Furthermore, as in Experiment 1 , there was an interaction between the factors of condition and stories $[F(1,38)=13.54$, $\mathrm{p}<.001]$. For "Simona," the summaries based upon the scrambled stories were not rated significantly different from the summaries based upon in tact stories (a difference of .61 with a standard error of .54), while this difference was large (1.50) and statistically reliable for "Rinaldo."

The rating data, therefore, not only confirm the sorting results of Experiment 1 with somewhat better statistical reliability, but they are also in full agreement with the experimental predictions. Nevertheless, one could argue that we have merely shown that summaries from scrambled stories do not differ from summaries of normal stories in some of the comparisons, not that either type story produces good summaries. The summaries might simply be all equally poor, irrespective of experimental conditions. A mean score of 3.4 on a 7-point scale does not tell us very much, because we do not know how high subjects would rate a really "ideal" summary. We therefore conducted Experiment 3.

\section{EXPERIMENT 3}

For our purposes, an ideal summary is one that correctly contains all major story elements within the 60- to 80-word length restriction imposed here. We are not concerned, however, with style and spelling errors. Thus, Boccaccio's own summaries of the two stories, or summaries constructed by the experimenters, might be rated high by our subjects not because of their structural qualities, but simply because their grammar is correct and their style is distinctly different from that of our experimental subjects. Hence, we needed "ideal" summaries written by the same population as the other summaries that were to be rated. To obtain such ideal summaries, we had subjects read each story (without restricting reading time), and then supplied them with an outline of the story which they were supposed to use in writing their summary. The "ideal" summaries thus obtained were then used together with the summaries from Experiment 1 in an additional rating experiment.

\section{Method}

Subjects. Twelve students participated in the summarization part of the experiment as partial fulfillment of a course requirement. An additional 20 subjects were paid for participating in the rating part of the study.

Procedure. In the summarization experiment, the subjects received instructions identical to those in Experiment 1 . In addition, before starting to summarize a story, they were handed an outline of the story (corresponding to Tables 1 and 2) and were asked to "use these statements in constructing your summary. You must include the meaning expressed in each of these statements in your summary. Do not attempt to reproduce the statements word for word; rather, use your own words to express their meaning." The order of stories was again counterbalanced. No warm-up story was used.

In the rating experiment, the instructions were the same as in Experiment 2. The procedure was the same too, except that subjects worked with sets of 15 summaries from each story. Three summaries were randomly selected from the "ideal" summaries; three from the free reading, natural order summaries three from free reading, scrambled order; three from restricted reading, natural order; and three from restricted reading, scrambled order. Thus, the design of this experiment was a complete within-subjects design.

\section{Results}

The results of the rating are also shown in Table 3 . An analysis of variance was conducted excluding the "ideal" summaries, in order to determine whether the results of Experiment 2 were replicated. All factors that were significant in Experiment 2 were significant again, except for one of the interactions. [Specifically, $F(1,19)=33.88$ for free vs. restricted reading time; $F<1$ for stories; $F(1,19)=40.92, p<.001$, for natural vs. scrambled order; $F(1,19)=11.14, p<.001$, for the Stories by Condition interaction; but $F(1,19)=1.72$, $\mathrm{p}=.210$, for the Reading Time by Condition interaction.] Thus, the overall pattern of results in Experiments 2 and 3 are quite comparable.

The main question of interest in Experiment 3 was how good the subjects' summaries were in comparison with the "ideal" summaries. For this purpose, a second analysis of variance of the data was conducted, this time 
including the "ideal" ratings. The two factors were stories and condition (ideal, natural, and scrambled for both free and restricted reading times). The latter factor was significant statistically $[F(4,76)=39.04$, $\mathrm{p}<.001]$, as well as the Stories by Condition interaction $[F(4,76)=4.01, p<.001]$. An orthogonal comparison between the ideal ratings and the natural order, free reading time ratings yielded $F(1,76)=2.58$, $p=.110$. Thus, the best summaries were not significantly worse than the "ideal" ones. Surely, this difference might become significant if more ratings were collected, and we do not want to conclude that the summaries subjects write with an outline are no better than those without one. The only point to be made is that the overall quality of the summaries, at least under the most favorable conditions (free reading, natural order), was reasonably good. Thus, the results reported in Experiments 1 and 2 and replicated here do not simply reflect a general inability of the subjects to compose acceptable summaries.

\section{DISCUSSION}

It appears that readers can summarize stories in which the paragraphs are presented out of order remarkably well; perfectly, in fact, when the story is a wellstructured one. We conclude that the reader's story schema permits him to reorganize the scrambled story, if that story corresponds to his schema. If the correspondence is not close enough, the restructuring of scrambled stories is not completely successful (especially when not enough time is allowed to read them), and the summaries become discriminably different from those written for intact stories.

The reading and writing times reported above support the argument that the macrostructure of a story is formed during reading, as part of the comprehension process, and not merely when one is asked to summarize the story. Subjects took $23 \%$ more time to read the scrambled stories than the normal stories, but there was no indication that the scrambled stories were harder to summarize. Indeed, averaged over the two experimental conditions, subjects took slightly less time to summarize scrambled stories than normal stories. This result enables us to locate the extra processing required by scrambled stories at the point of comprehension, rather than in the response phase.

Subjects understood "Simona" (as indicated by the summaries they wrote), whether it was presented in natural or in scrambled order, when reading time was free and with only a slight deterioration when it was restricted. On the other hand, reading "Rinaldo" in scrambled order produced problems. These problems were minor when reading time was free, but more serious when not enough reading time was permitted. A related tendency was observed by Kintsch and van Dijk (1975), where subjects agreed more among each other about what to write in the summaries for
"Simona" than for "Rinaldo," precisely what was concluded earlier on the basis of the episode structure of the stories. Obviously, there are many other differences between "Simona" and "Rinaldo" which are confounded with the structural differences emphasized here. Further research with different stories will be needed before the between-stories differences observed here can be fully attributed to differences in structure, as we have suggested.

Showing that subjects comprehend well-structured stories even when their paragraphs are presented out of order does not, of course, prove the quite elaborate theory of story comprehension outlined in the introduction to this paper. However, this rather surprising ability does need to be explained, and our theory easily accounts for it.

Indeed, the evidence suggests that subjects reorder scrambled stories while reading them, so that the end product of comprehension is a macrostructure for the story that is not discriminably different from the macrostructure derived from the same story in its natural order. At least, this is the case when the story schema fits the actual story well. If the story is less predictable from the knowledge of the schema, this restructuring of scrambled texts is not completely successful. The results from the experiments with scrambled stories confirm, therefore, a crucial point of the theory, namely, that the formation of macrostructures is an integral part of the comprehension process and occurs during reading, not when the story is recalled or summarized.

\section{REFERENCES}

Bartlett, F. C. Remembering. Cambridge: Cambridge University Press, 1932.

Boccaccio, G. [The decameron] (G. H. McWilliam, trans.). London: Penguin Books, 1972.

Halliday, M. A. K., \& Hasan, R. Cohesion in English. London: Longman, 1976.

Kintsch, W., \& van Dijk, T. A. Comment on se rapelle et on résume des histoires. Langages, 1975, 40, 98-116.

MANDleR, J. M., \& Johnson, N. S. Remembrance of things parsed: Story structure and recall. Cognitive Psychology, 1977, 9, 111-151.

Rosenberg, S., \& LAMBert, W. E. Contextual constraints and the perception of speech. Journal of Educational Psychology, 1974, 102, 178-180.

Rumelhart, D. E. Notes on a schema for stories. In D. G. Bobrow \& A. M. Collins (Eds.), Representation and understanding. New York: Academic Press, 1975.

Schank, R. C. SAM-A story understander. Technical Report No. 43, Department of Computer Science, Yale University, 1975.

ThORNDYKE, P. W. Cognitive structures in comprehension and memory of narrative discourse. Cognitive Psychology, 1977, 9, 77-110.

van Dijk, T. A., \& Kintsch, W. Cognitive psychology and discourse: Recalling and summarizing stories. In W. U. Dressler (Ed.), Trends in textlinguistics. New York-Berlin: de Gruyter, in press.

(Received for publication January 4, 1977; revision accepted June 24,1977 .) 